Jochen Brenneisen*, Carlo Wentzel, Farokh Karwan, Olaf Dössel and Axel Loewe

\title{
Fluid dynamics in the human heart: Altered vortex formation and wash-out in mitral regurgitation simulations
}

\begin{abstract}
Mitral regurgitation alters the flow conditions in the left ventricle. To account for quantitative changes and to investigate the behavior of different flow components, a realistic computational model of the whole human heart was employed in this study. While performing fluid dynamics simulations, a scalar transport equation was solved to analyze vortex formation and ventricular wash-out for different regurgitation severities. Additionally, a particle tracking algorithm was implemented to visualize single components of the blood flow.

We confirmed a significantly lowered volume of the direct flow component as well as a higher vorticity in the diseased case.
\end{abstract}

Keywords: CFD, fluid dynamics simulation, mitral regurgitation, flow pattern.

https://doi.org/10.1515/cdbme-2021-2051

\section{Introduction}

Mitral regurgitation (MR) is of increasing prevalence in Europe [1]. Caused by a diseased mitral valve (MV) that is not closing properly, blood regurgitates from the left ventricular chamber into the left atrium (LA). The reduced forward stroke volume may limit physical capabilities and lead to long term overload of the heart.

As computer models of the human heart are a powerful tool to investigate disease phenomena and to non-invasively quantify many measures, we simulated the altered blood flow in the left heart. Visualization of flow patterns is a powerful simulation-based tool to reveal disease-induced flow alterations. Thus, in this work we evaluated altered flow

\footnotetext{
*Corresponding author: Jochen Brenneisen: Institute of Biomedical Engineering, Karlsruhe Institute of Technology (KIT), Kaiserstraße 12, 76131 Karlsruhe, Germany, publications@ibt.kit.edu

Carlo Wenzel, Farokh Karwan, Olaf Dössel and Axel Loewe: Institute of Biomedical Engineering, Karlsruhe Institute of Technology (KIT), Karlsruhe, Germany
}

patterns in the left ventricle (LV) caused by different severities of a regurgitant mitral valve as well as the wash-out of the left ventricular chamber.

\section{Methods}

\subsection{Model generation}

The basis of this work is a patient-specific healthy heart geometry that was created from MRI scans and discretized using finite element techniques. Focusing on the mitral valve, the model comprises the LA and LV as well as prolonged tubes that idealize the four pulmonary veins and the aorta for steady state flow conditions (see Figure 1).

For the fluid dynamic simulations, the movement of the endocardial surfaces and the in- and outlet pressure over time were taken into account as boundary conditions (BC). Applying our mechanical simulation framework [2], the BC were obtained through a whole-heart mechanical simulation. The closed-loop circulatory system model [3] supplied the chamber pressure curves. In this way, a unidirectional coupling of cardiac continuum mechanics to the fluid dynamics solver was established [4]. To model the moving geometry caused by the movement of endocardial surfaces, an Arbitrary Lagrangian Eulerian (ALE) description was implemented [5].

To resemble flow characteristics, all heart valves were represented by a planar model based on a porous material law [6]. The variation of this zone's permeability over time models the valve characteristics in a compact way and is controlled depending on flow. By solving the Navier-Stokes equations (NSE), the pressure distribution and the velocity field across the left part of the heart geometry were computed. Modeling blood as an incompressible Newtonian fluid, the NSE condenses to the following partial differential equation:

$$
\rho\left(\frac{\partial u_{i}}{\partial t}+u_{j} \frac{\partial u_{i}}{\partial x_{j}}\right)=-\frac{\partial p}{\partial x_{i}}+\mu\left(\frac{\partial^{2} u_{i}}{\partial x_{j} \partial x_{j}}\right)
$$

where $\rho=1055 \mathrm{~kg} \mathrm{~m}^{-3}$ denotes the density and $\mu=$ $0.004 \mathrm{~kg} \mathrm{~m}^{-1} \mathrm{~s}^{-1}$ the dynamic viscosity of blood, while $u_{i}$ is the fluid velocity in the direction of $x_{i}$. 
All simulations were performed in the open-source software package openFOAM v1912 [7].

\subsection{Disease modeling}

Daub et al. [6] showed for an idealized heart geometry that the planar MV model is able to reproduce physiological flow fields. The Darcy-Forchheimer law [8] is used to quantify the pressure drop across the MV by adjusting the permeability of the porous zone.

Extending this approach, we model a diseased valve based on the porous zone implementation in this study. To model pathology, an elliptic hole was added in the posterior leaflet in medial direction. By including holes of different diameter in the MV, mitral regurgitation with different severities was modelled by different effective regurgitant orifice areas according to European Society of Cardiology (ESC) guidelines [1]. The hole diameter and the corresponding severity can be found in Table 1 .
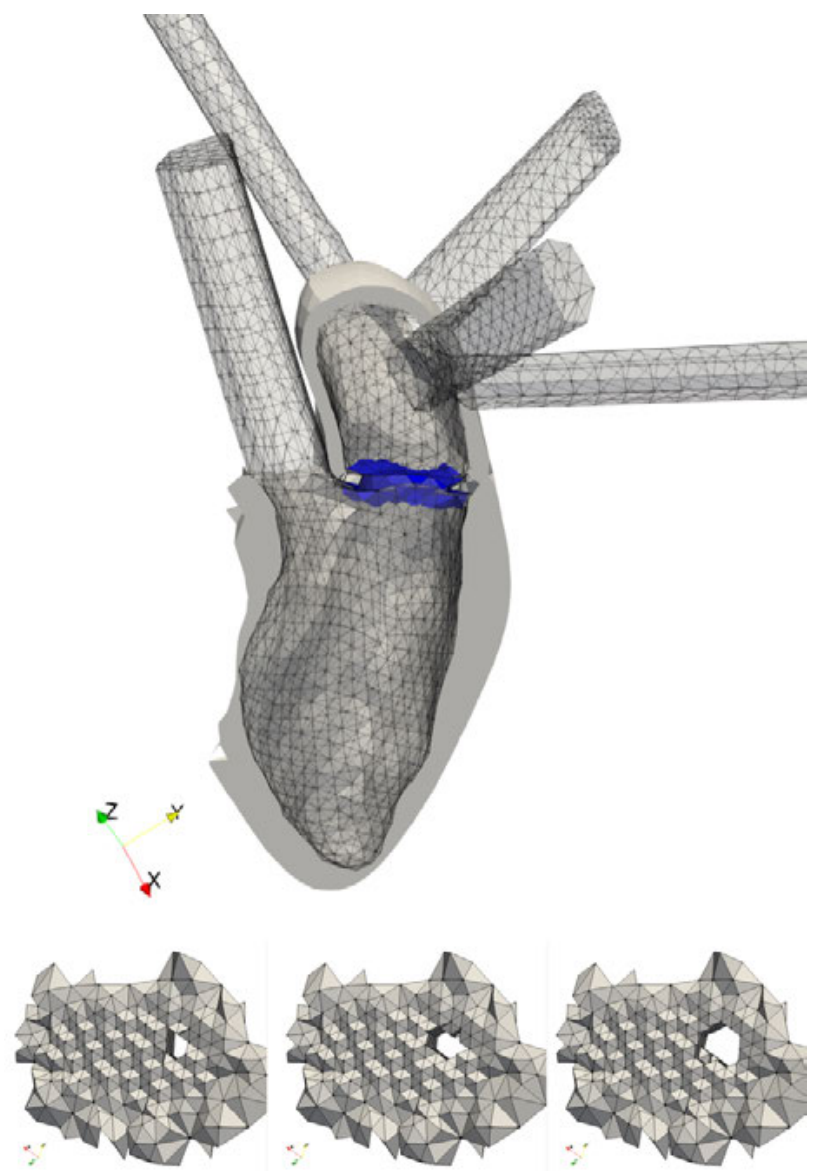

Figure 1: Top: Finite element model of the left heart. Mitral valve zone marked in blue. Bottom: Porous zones that model mitral regurgitation with severity mild, moderate and severe from left to right.
Table 1: MR severity according to ESC guidelines [1].

\begin{tabular}{llll}
\hline MR severity & mild & moderate & severe \\
\hline $\begin{array}{l}\text { Effective Regurgitation } \\
\text { Orifice Area }\left(\mathrm{mm}^{2}\right)\end{array}$ & $<20$ & $20-39$ & $\geq 40$ \\
Regurgitant volume $(\mathrm{ml})$ & $<30$ & $30-59$ & $\geq 60$ \\
\hline
\end{tabular}

\subsection{Evaluation methods}

\subsubsection{Scalar transport}

To evaluate the fraction of the blood volume that was initially located in the LV and remains there, a passive scalar transport equation was implemented. The scalar $\psi$ therefore can be considered as a measure of local concentration of initial blood volume. It can be calculated using the following equation:

$$
\frac{\partial \psi}{\partial t}+\nabla \cdot(\boldsymbol{U} \psi)-\Delta(D \psi)=\mathbf{0}
$$

where $\psi$ is computed based on the velocity field $U$ and the diffusion coefficient $D=10^{-10} \mathrm{~m}^{2} \mathrm{~s}^{-1}$ with the initial value $\psi\left(\mathrm{x}, \mathrm{t}_{0}\right)=1$ for all cells located in the LV and $\psi\left(\mathrm{x}, \mathrm{t}_{0}\right)=0$ for all others.

\subsubsection{Blood flow component analysis}

Introduced by Bolger et al. [9], the separation of blood flow in four different flow components is a common method to quantify component fractions and to visualize trajectories.

The blood volume passing through the LA is composed of the 'direct flow' (DF) that directly leaves the LV in the same cycle and the 'retained inflow' (RI) that remains in the LV during the concerning cycle. The blood component that is already located in the LV at the beginning of the heart cycle and then ejected through the aorta is named 'delayed ejected flow' (DEF), whereas the volume remaining in the LV is the 'residual volume' (ReV). Considering the back flow (BF) that regurgitates through the $\mathrm{MV}$ as a fifth component, the visualization of the components can be seen in Figure 2.

\subsubsection{Particle Tracking}

In a post-processing step, a particle tracking algorithm was implemented to compute the position of an arbitrary single particle in time and in space. Given the spatially and temporally resolved velocity field $\mathrm{U}$, the tracking is computed based on the integration of the concerning movement equation. A number of $n \approx 15000$ particles were analyzed. 


\section{Results}

Altered flow patterns (particular in end-diastole) and wash-out fractions were observed in the diseased geometry. Compared to the healthy case (Figure 3A), the blood spread deeper

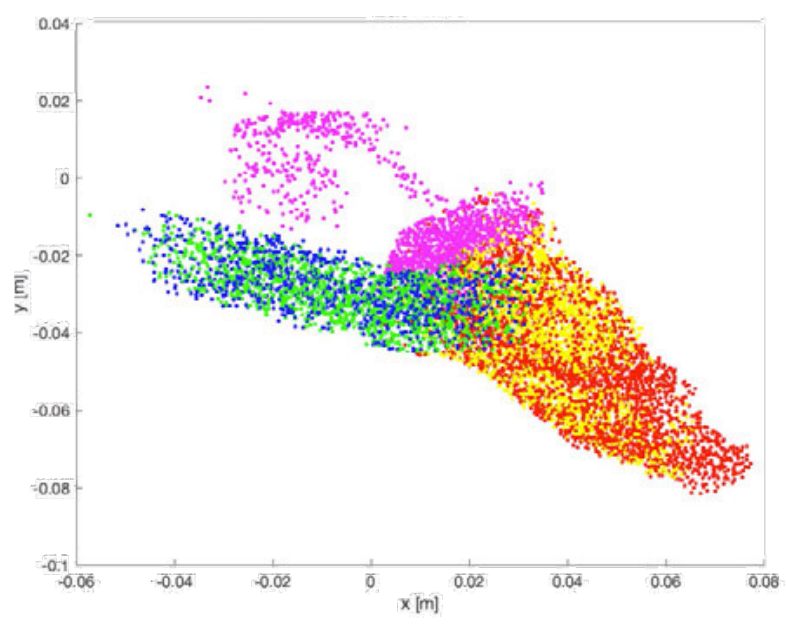

Figure 2: Visualization of blood flow components (moderate MR) in end-diastolic state. Color codes for the flow components: DF (green), RI (yellow), DEF (blue), ReV (red) and BF (pink).
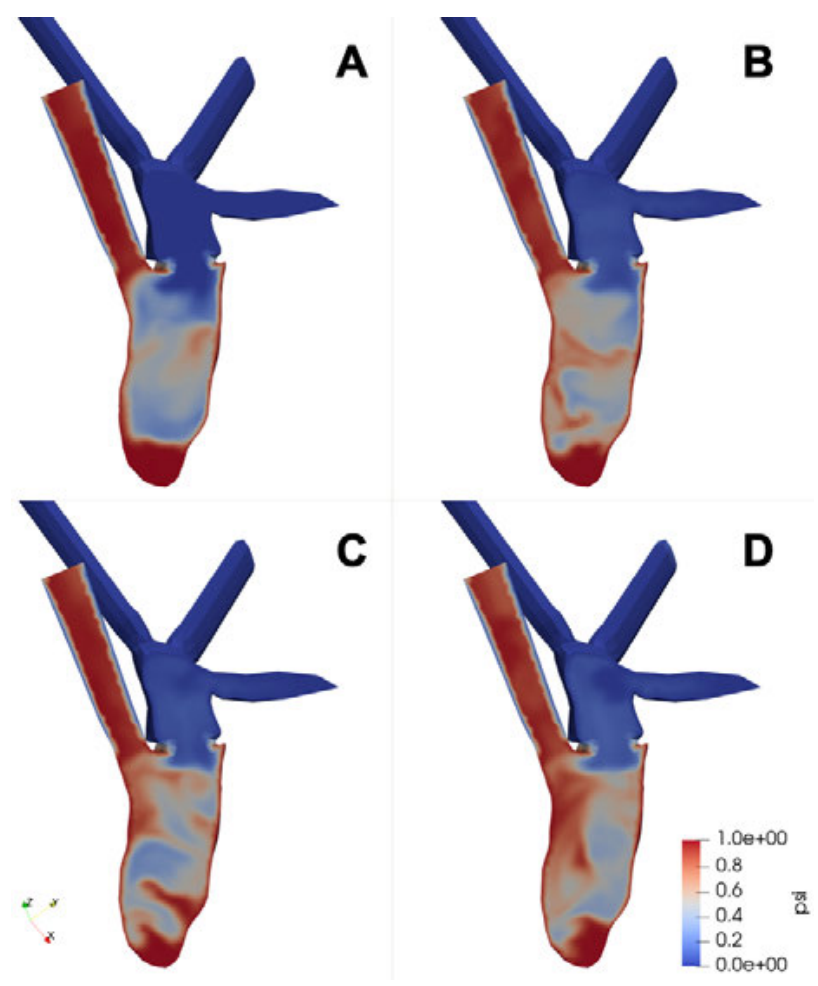

Figure 3: Long axis slices of healthy heart $(A)$ and mild, moderate and severe MR (B-D). Time: Atrial contraction of the second cycle $\mathrm{t}=1.39 \mathrm{~s}$. towards the apex in the diseased case (Figure 3B-D) while the blood in the LV itself was less washed out, as the distribution of the scalar $\psi$ in the figure indicates. The vorticity in the diseased case was higher throughout all severity grades.

With increasing MR severity, a non-linear behavior of remaining initial blood volume was observed. Comparing four heart cycles, depending on the cardiac phase and MR severity, phases with de- and increased values were observed. E.g., for moderate mitral regurgitation, the ventricular wash-out was up to $6 \%$ lower than in the healthy control case in the second heart cycle (Figure 4).

The DF flow component was decreased for all MR severity stages. Quantitative results, exemplary for moderate MR are shown in Table 2. For this case, the regurgitant blood caused a DF decrease of $18 \%$. All other blood flow components varied slightly around $\pm 2 \%$. Already for a moderate MR, the regurgitant volume was around $15 \%$ of LV volume.

Evaluating the results of the particle tracking algorithm confirmed the previous results. The direct flow was reduced by more than $10 \%$, accounting for the slower wash-out. The visualization of all five blood flow components confirms the high concentration of ReV blood in the apex as well as a low mixture of $\mathrm{ReV}$ blood with blood in the lower part of the LV that entered the LV in the current cycle (Figure 4).

Table 2: Blood flow components in MR compared to healthy case.

\begin{tabular}{llllll}
\hline & $\begin{array}{l}\text { direct } \\
\text { flow }\end{array}$ & $\begin{array}{l}\text { retained } \\
\text { inflow }\end{array}$ & $\begin{array}{l}\text { delayed } \\
\text { ejection }\end{array}$ & $\begin{array}{l}\text { residual } \\
\text { volume }\end{array}$ & $\begin{array}{l}\text { back } \\
\text { flow }\end{array}$ \\
\hline healthy & $35.3 \%$ & $16.0 \%$ & $21.9 \%$ & $26.8 \%$ & \\
$\begin{array}{l}\text { moderate } \\
\text { MR }\end{array}$ & $17.3 \%$ & $17.5 \%$ & $24.3 \%$ & $25.5 \%$ & $15.4 \%$ \\
\hline
\end{tabular}

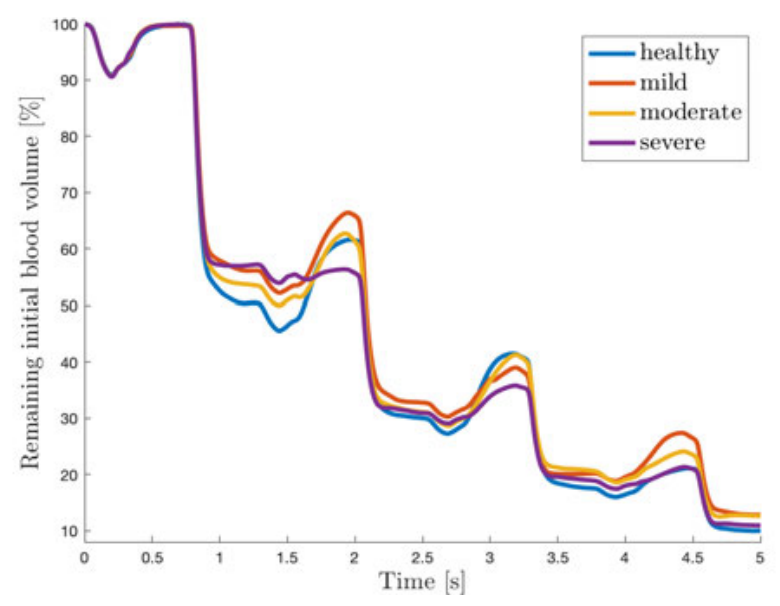

Figure 4: Scalar $\psi$ as measure for local concentration of initial blood volume that remains in the LV over a time course of four heart cycles. Different degrees of mitral regurgitation. 


\section{Discussion and Outlook}

Comparing the three severity stages presented in the long axis view in Figure 3, a clear distinction between the severity stages is hard to define. Additionally, the visualized long axis views only show a single plane of the whole LV volume. Taking into account the non-linear dependency between the degree of regurgitation and the increase of the $\mathrm{ReV}$ time courses in Figure 4 additionally shows that only analyzing a mean value across the whole LV does not account for local variations in altered flow conditions. Therefore, the presented measures may not be sufficient to fully quantify the resulting flow alterations. In addition to the presented methods, 2D short axis slices of the LV could be analyzed regarding their vorticity behavior. An analysis and quantification of vortex structures in different heights could be carried out to account for vorticity location maps. This method could also be used as a measure for the immersion depth of the ventricular jet. This may link to the work of Collia et al. [10] that clearly associated a prolapsed MV with a higher amount of residual volume close to the apex as expected from a lower jet energy. However, a literature comparison will remain challenging, as the classification of MR severities is only based on upper and lower limits instead of exact values, as shown in Table 1.

Another point to further investigate is the capability of the MV model. As different severity stages are only distinguished by a de- or increased Effective Regurgitation Orifice Area, the elliptic symmetric hole in the medial direction of the posterior leaflet may not fully cover all MR effects. Implementing a 3D parametrical valve model parametrized on the basis of measurement data will serve as a comparison in further studies.

The method of using a scalar transport to account for blood concentrations however delivered accurate results. A comparison to literature 4D MRI measurement data [11] showed good accordance with our simulation results of the healthy heart geometry with deviations around $\pm 2 \%$ for the single flow components.

\section{Conclusion}

Modeling the MV with a simple planar valve model delivered accurate results in healthy heart models [6]. Extending this approach to patients suffering from MR clearly revealed an alteration of blood flow patterns as well as altered ventricular wash-out fractions and significantly reduced direct flow. Additionally, the implementation of a particle tracking algorithm for post-processing the CFD simulation data enabled visualization of the different flow components and analysis of the start and end positions of the concerning trajectories. However, the full quantitative analysis and comparison of different severity stages remains challenging and further analysis tools have to be implemented to fully characterize LV vorticity. In conclusion, this work and the implemented methods build a basis to characterize vortex structures in mitral valve disease in future studies.

\section{Author Statement}

Research funding: This work was funded by the state of Baden-Württemberg. Conflict of interest: Authors state no conflict of interest. Informed consent: Informed consent has been obtained from all individuals included in this study. Ethical approval: The study was approved by the local IRB.

\section{References}

[1] Lancellotti P, Moura L, Pierard LA, Agricola E, Popescu BA, Tribouilloy $C$, et al. European Association of

Echocardiography recommendations for the assessment of valvular regurgitation. Part 2: mitral and tricuspid regurgitation (native valve disease). Eur J Echocardiogr 2010;11:307-32

[2] Fritz T, Wieners C, Seemann G, Steen H, Dössel O. Simulation of the contraction of the ventricles in a human heart model including atria and pericardium. Finite element analysis of a frictionless contact problem. Biomech Model Mechanobiol 2014, 13:627-641

[3] Gerach T, Schuler S, Fröhlich J, Lindner L, Kovacheva E, Moss $R$ et al. Electro-Mechanical Whole-Heart Digital Twins: A Fully Coupled Multi-Physics Approach. Mathematics 2021(9): 1247

[4] Daub A. Numerical Haemodynamics in the Human Heart. Karlsruhe: KIT Scientific Publishing; 2018

[5] Souli M, Zolesio JP. Arbitrary Lagrangian-Eulerian and free surface methods in fluid mechanics. Comput Methods Appl Mech Eng 2001;191:451-466

[6] Daub A, Kriegseis J, Frohnapfel B. Replication of left ventricular haemodynamics with a simple planar mitral valve model. Biomed Eng-Biomed Tech 2020;65(5):595-603

[7] Weller HG, Tabor G, Jasak H, Fureby C. A tensorial approach to computational continuum mechanics using object-oriented techniques. Comput Phys 1998,12(6)

[8] Irmay S. On the theoretical derivation of Darcy and Forchheimer formulas. EOS, Trans Am Geophys Union 1958;39:702-707

[9] Bolger AF, Heiberg E, Karlsson M, et al. Transit of blood flow through the human left ventricle mapped by cardiovascular magnetic resonance. J Cardiovasc Magn Reson 2007, 9(5):741-747

[10] Collia D, Zovatto L, Pedrizzetti G. Analysis of mitral regurgitation by computational fluid dynamics. APL Bioeng. 2019,3(3):036105

[11] Eriksson J, Bolger AF, Ebbers T, Carlhäll CJ. Fourdimensional blood flow-specific markers of LV dysfunction in dilated cardiomyopathy. Eur Heart J 2013,14:417-424 